\title{
Erratum to: The societal costs of dementia in Sweden 2012 - relevance and methodological challenges in valuing informal care
}

Anders Wimo ${ }^{1,2,3^{*}}$, Linus Jönsson ${ }^{1}$, Laura Fratiglioni ${ }^{2}$, Per Olof Sandman ${ }^{4,5,6}$, Anders Gustavsson', Anders Sköldunger ${ }^{2}$ and John Lennarth Johansson ${ }^{7}$

\section{Erratum}

During production of the original article [1], the line referring to "Informal Care" was omitted from Table 3. It should have been mentioned between "Total social care sector" and "Indirect costs".

\begin{abstract}
Author details
${ }^{1}$ Aging Research Centre, Department of Neurobiology, Care Sciences and Society (NVS), Karolinska Institutet and Stockholm University, Stockholm, Sweden. ${ }^{2}$ Division of Neurogeriatrics, Department of Neurobiology, Care Sciences and Society (NVS), Karolinska Institutet, Huddinge, Stockholm, Sweden. ${ }^{3}$ Centre for Research \& Development, Uppsala University/County Council of Gävleborg, Gävle, Sweden. ${ }^{4}$ Division of Caring Sciences, Department of Neurobiology, Care Sciences and Society (NVS), Karolinska Institutet, Stockholm, Sweden. ${ }^{5}$ Department of Nursing, Umeå University, Umeå, Sweden. ${ }^{6}$ Department of Health Sciences, Luleå University of Technology, Luleå, Sweden. ${ }^{7}$ Stockholm Gerontology Research Centre, Stockholm, Sweden
\end{abstract}

Received: 19 December 2016 Accepted: 9 February 2017

Published online: 27 February 2017

\section{Reference}

1. Wimo A. The societal costs of dementia in Sweden 2012 - relevance and methodological challenges in valuing informal care. BMC Alzheimer's Research \& Therapy. 2016;8:59. doi:10.1186/s13195-016-0215-9.

* Correspondence: anders.wimo@ki.se

${ }^{1}$ Aging Research Centre, Department of Neurobiology, Care Sciences and Society (NVS), Karolinska Institutet and Stockholm University, Stockholm, Sweden

2Division of Neurogeriatrics, Department of Neurobiology, Care Sciences and Society (NVS), Karolinska Institutet, Huddinge, Stockholm, Sweden 
Table 3 Total societal costs of dementia in Sweden in 2012 (base option)

\begin{tabular}{|c|c|c|c|}
\hline & Cost (million SEK) $)^{a}$ & Per PWD, SEK ${ }^{\mathrm{a}}$ & Proportion \\
\hline \multicolumn{4}{|l|}{ Medical care sector (county councils) } \\
\hline Hospital care & 276 & & $0.4 \%$ \\
\hline Emergency room visits & 264 & & $0.4 \%$ \\
\hline Outpatient care visits (specialists) & 57 & & $0.1 \%$ \\
\hline Primary care physician visits & 360 & & $0.6 \%$ \\
\hline Other outpatient care (e.g., rehabilitation) & 589 & & $0.9 \%$ \\
\hline Drug use & 1120 & & $1.8 \%$ \\
\hline Diagnostic work ups & 240 & & $0.4 \%$ \\
\hline Total medical care sector & 2904 & 18,382 & $4.6 \%$ \\
\hline \multicolumn{4}{|l|}{ Social care sector (municipalities) } \\
\hline Institutional care (permanent) & 38,199 & & $60.7 \%$ \\
\hline Short-term respite care & 2364 & & $3.8 \%$ \\
\hline Day Care & 989 & & $1.6 \%$ \\
\hline Home services & 7372 & & $11.7 \%$ \\
\hline Dementia nurses & 338 & & $0.5 \%$ \\
\hline Total social care sector & 49,262 & 311,783 & $78.3 \%$ \\
\hline Informal care & 10,636 & 67,318 & $16.9 \%$ \\
\hline Indirect costs & 118 & 744 & $0.2 \%$ \\
\hline Total ${ }^{b}$ & 62,920 & 398,226 & $100.0 \%$ \\
\hline
\end{tabular}

PWD Persons with dementia, SEK Swedish krona

${ }^{\mathrm{a}} 1 €$ corresponds to SEK 8.77 and 1 US\$ to SEK 6.96

${ }^{\mathrm{b}}$ Discrepancies due to rounding 